\title{
System Autonomy for Space Traffic Management
}

\author{
Sreeja Nag*, David Murakami ${ }^{\dagger}$, Miles Lifson*, Parimal Kopardekar ${ }^{\dagger}$ \\ *NASA Ames Research Center and Bay Area Environmental Research Institute, Moffet Field, CA, USA \\ ${ }^{\dagger}$ NASA Ames Research Center, Moffet Field, CA, USA \\ Email: Sreeja.Nag@nasa.gov, David.D.Murakami@nasa.gov
}

\begin{abstract}
This paper proposes an initial architecture for a Space Traffic Management (STM) system, based on open Application Programming Interfaces (APIs) and drawing on previous work by NASA to develop an architecture for lowaltitude Unmanned Aerial System Traffic Management (UTM). The authors explore how autonomy could be used to enhance an STM system, and how constraints inherent in STM complicate and challenge certain applications of autonomy. We conceptually explore how autonomy could be used within an STM architecture, with multiple non-authoritative catalogs of resident space objects, and to determine which of two spacecraft moves to prevent an impending conjunction between them. NASA Ames Research Center (ARC) is developing a software research environment for STM, along with a physical laboratory and visualization space. We invite STM stakeholders to collaborate in our infrastructure, to help inform the design of the proposed STM architecture, and to participate in the refinement and validation of its concept of operations using the software research platform.
\end{abstract}

Index Terms-Space Traffic Management, Collision Avoidance, Space Situational Awareness, Autonomy, Space Policy

\section{INTRODUCTION}

As ever larger numbers of spacecraft seek to make use of Earth's limited orbital volume in increasingly dense orbital regimes, greater coordination will become necessary to ensure these spacecraft are able to operate safely while avoiding physical collisions, radio-frequency interference, and other hazards. While efforts to date have focused on improving space situational awareness (SSA) and enabling operator to operator coordination, there is growing recognition that a broader system for space traffic management (STM) is necessary. In June 2018, President Trump signed Space Policy Directive 3, ordering the development of "standards and protocols for creation of an open architecture data repository to improve SSA data interoperability and enable greater SSA data sharing" [1]. While significant technical and regulatory progress will be necessary to develop an operational STM system, the viability of any such system will hinge on its ability to facilitate most of the necessary synchronization and planning at an automated machine to machine (M2M) level.

NASA Ames Research Center (ARC) proposes an STM system architecture and concept of operations based on APIdefined interfaces between data and service suppliers, consumers of STM services, and regulators within a broader U.S. STM ecosystem. Critically, the well-defined interfaces between the different entities enables the application of autonomy to problems that have historically required significant hu-

Funding - NASA Aeronautics Research Mission Directorate and NASA HQ man participation, due to either the need for manual interfaces between actors or existence of unsupported non-standard interactions. This paper presents several STM-related system characteristics that might complicate automation, briefly proposes a conceptual STM architecture and applies it to an example collision avoidance case, discusses potential roles for systemwide autonomy within STM, and describes initial thinking on ways to address the challenges to autonomy identified. NASA ARC is developing a software-based research platform that will allow for testing of these strategies and refinement of the proposed system concept of operation.

\section{Challenges for STM Autonomy}

There are several dimensions of the STM architecture, which challenge the application of system autonomy.

\section{A. The STM system must accommodate safe operations in the presence of non-participants}

An STM system will need to be designed to accommodate non-participating spacecraft, either those from outside the jurisdiction of the STM system or who refuse to participate. International law affirms the right of all countries to use space, and even if policy-makers choose to open a proposed U.S. system to other nations on a voluntary basis, the U.S. would lack any legal authority to mandate participation. Meanwhile, existing spacecraft will need to deconflict with non-participants to avoid collisions. Similarly, while civil and commercial spacecraft may comply with an STM system, national security entities will be extraordinarily reluctant to disclose the positions or maneuvering of their unacknowledged spacecraft. While conjunctions between participating and nonparticipating spacecraft cannot be addressed using the STM system, standards could be developed to encourage information sharing.

\section{B. The STM system must allow owner/operators (O/Os) to} maintain operational control over their spacecraft

To be politically palatable, an STM system cannot compel $\mathrm{O} / \mathrm{Os}$ to cede operational control of their spacecraft to space traffic management service suppliers $(\mathrm{S} 3 \mathrm{~s})^{1}$ or a government entity. While some, particularly small $\mathrm{O} / \mathrm{Os}$, may be willing to do so, the system will need to be designed to accommodate

\footnotetext{
${ }^{1}$ The S3 concept is discussed further as part of the our proposed STM architecture in Section III. S3s serve as a link between O/Os and the rest of the STM system, providing services such as conjunction assessment and collision avoidance manuever development.
} 
negotiations between $\mathrm{O} / \mathrm{Os}$ and $\mathrm{S} 3 \mathrm{~s}$ and failure modes if an $\mathrm{O} / \mathrm{O}$ does not perform a maneuver correctly or refuses to do so. In practice, the split of responsibilities between an S3 and $\mathrm{O} / \mathrm{O}$ may vary based on the $\mathrm{S} 3$ and $\mathrm{O} / \mathrm{O}$. For instance, while a low sophistication $\mathrm{O} / \mathrm{O}$ might only want to maintain a go/nogo determination on potential collision avoidance maneuvers, a sophisticated operation might prefer to review and sometimes counter-propose collision avoidance maneuvers to its S3.

\section{The STM system must tolerate imperfect and private data}

Non-cooperative optical and radio-based measurement techniques used to locate and characterize space objects can determine object positions and enable propagation to future trajectories. However, they have limited predictive accuracy and cannot predict or assess maneuvers, particularly long-term low-thrust maneuvers like those conducted by satellites using electric propulsion. O/Os often have much better accuracy positional information for their own spacecraft than can be obtained non-cooperatively.

Some O/Os are reluctant to share this $\mathrm{O} / \mathrm{O}$ ephemeris information for proprietary reasons. GEO operators collaborated to develop a non-profit organization called the Space Data Association to provide a trusted legal and technical means to exchange proprietary orbital and radio-frequency information in a protected setting. With limited willingness to share information, the STM system must tolerate actors attempting to deconflict collisions without full information, and potentially with differing understanding of the states of their own spacecraft and those with which they are attempting to deconflict. The proposed architecture anticipates the existence of commercial providers of SSA observations who, depending on their business model, may not be willing to disclose observations publicly. One potential consequence of both $\mathrm{O} / \mathrm{O}$ reluctance to share higher-accuracy positional information and an open architecture is the presence of multiple competing or separate catalogs of resident space objects (RSOs). Proposals exist to achieve high resolution information through mechanisms other than $\mathrm{O} / \mathrm{O}$ ephemeris, including through independent GPS modules [2], but these solutions raise similar proprietary concerns for $\mathrm{O} / \mathrm{Os}$.

\section{Proposed Architecture For STM CONCEPT OF OPERATIONS}

NASA ARC is developing a concept for an automated and distributed space traffic management architecture, drawing on similar NASA work done to provide traffic management for small unmanned aircraft systems (UAS) operating at low altitudes [3] [4], and adopting the same guiding principle of "flexibility where possible and structure where necessary".

Our architecture proposes a strategy to accommodate growing space traffic volume safely and flexibly, as well as provide the technical means for a transition of STM authority to a civilian governmental entity. The architecture envisions an open-access software platform that connects data and service suppliers, consumers, and regulators via open and standard APIs. The platform would build on, rather than replicate coordination efforts within the SSA ecosystem, using existing standards for data message formats from organizations like the Consultative Committee for Space Data Systems (CCSDS), and wrapping rather than replacing existing integrations. The system seeks to provide the technical means to shift from a paradigm of manual integration between a limited number of major organizations, to one that provides low-friction means to disseminate and harness diverse, but valuable sources of STM-relevant information with minimal human intervention.

The architecture is designed to be developed through a series of technical capability levels (TCLs), beginning with a focus on safe operations of and interaction with small satellites in low Earth orbit. As the TCLs advance over time, STM capabilities would expand to include satellites of all sizes, all orbits, launch and reentry activities, and additional functions for radio-frequency interference, space weather, and laser operation. The system would be developed for U.S. supervised traffic, but could inform subsequent efforts to develop a more international system.

Key roles within the system include:

- The Spaceflight Information Management System (SIMS), which serves as a privileged connector between national regulatory authorities and STM, gathering information for regulatory purposes or pushing urgent alerts or orbital constraint information as directed by regulators. National authorities such as the Federal Communications Commission (FCC), Federal Aviation Administration (FAA), National Telecommunications and Information Administration (NTIA), Department of State, NASA or NOAA, are expected to communicate with the international regulatory authorities, such as International Telecommunications Union (ITU), Inter-Agency Space Debris Coordination Committee (IADC) or United Nations Office for Outer Space Affairs (UNOOSA), separately.

- Owner/Operators (O/O), who own and fly satellites participating in the STM architecture.

- STM Service Suppliers (S3), who act as a concierge providing STM \& compliance services to O/Os. S3s serve as a link to the broader STM ecosystem and procure services on behalf of $\mathrm{O} / \mathrm{Os}$.

- Space Situational Awareness Suppliers (SSA), who are responsible for acquiring and fusing sensor observations and cooperative tracking data from $\mathrm{O} / \mathrm{O}$ s to generate and maintain a catalog of space objects.

- Conjunction Assessment Suppliers (CAS), who are responsible for screening objects against SSA catalogs for potential conjunctions, as well as verifying collision avoidance maneuvers proposed by S3s.

- Supplemental Data Suppliers (SDS), who provide other relevant data and services. Examples might include precision atmospheric modeling data to reduce errors in spacecraft orbit propagation or space weather warnings.

Each of these roles have associated standard APIs with required and optional fields. Software written to interface with 


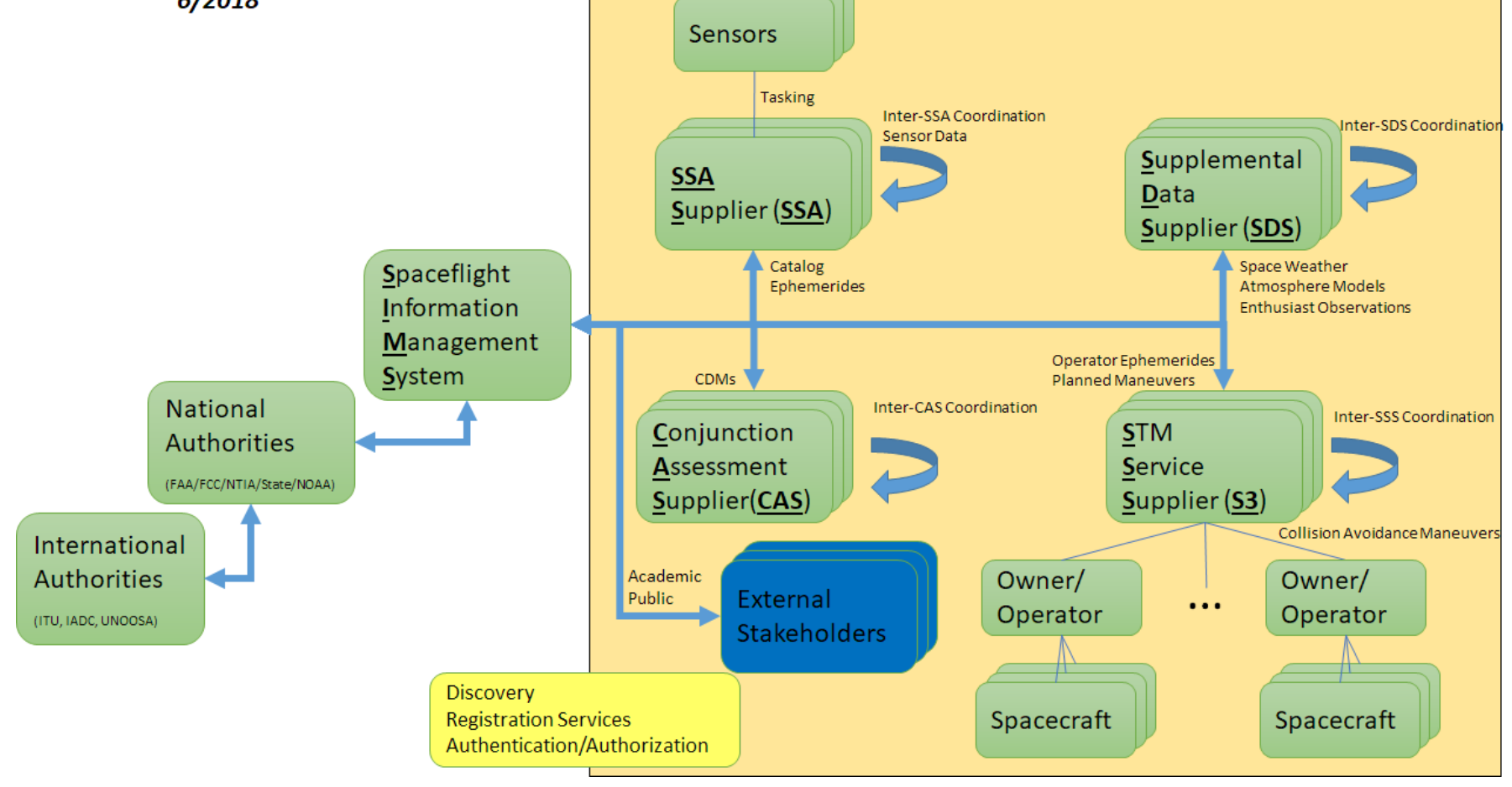

Fig. 1. A notional outline of the proposed STM architecture.

one particular organization can be easily reused with little or no modification to interface with any other performing the same role. These roles are described in functional terms, but the STM architecture makes no assumptions about the type of actor who would provide a given service (government, nonprofit, or commercial) or regarding the separation of roles across actors. Multiple roles might be fulfilled by a single conceptual or legal entity. For instance, a large $\mathrm{O} / \mathrm{O}$ who flies many satellites might choose to act as its own S3, or contract with an outside provider. Similarly, a single company might provide both SSA and CAS services. Space Policy Directive 3 directs that a civilian STM authority should continue to provide free basic SSA and SSA services, consistent with those currently provided by U.S. Strategic Command. The proposed architecture system would accommodate these services, while also allowing the participation of commercial and non-profit entities, with varying structures and business/service models.

A white paper describing the architecture and concept of operations in greater detail is under development. NASA ARC is developing a software research platform featuring an implementation of the STM architecture concept to assess test cases and refine it in partnership with industry and academia.

\section{Application of the StM Architecture to COLLISION AVOIDANCE}

This section walks through an operational example of a collision avoidance maneuver within the proposed STM architecture, to exemplify the roles of the various actors involved within $\mathrm{STM}^{2}$. This example involves a conjunction and subsequent collision avoidance maneuver between two active satellites. The first, SpaceCube, is a small satellite without propulsion operated by the University of Spaceland, which contracts with Service4Less, Inc., a commercial S3. The other is SatCom1, operated by SatComCo, which also serves as its own S3. For the sake of this example, assume both $\mathrm{O} / \mathrm{Os}$ have well-known positions for their own satellite. It is worth nothing that apart from initial setup work, the complex interactions described in the following occur largely automatically, via APIs in the STM architecture. Manual intervention occurs primarily at the O/O level, which holds the ultimate responsibility for safety.

\section{A. Registration}

Prior to the start of the example, U. of Spaceland registered with Service4Less, providing its contact information and position and meta-data for SpaceCube. Service4Less then updated the system-wide STM registry to indicate it provides S3 services to U. of Spaceland for SpaceCube. At agreed upon intervals, U. of Spaceland provides updated O/O ephemeris to Service4Less based on the more precise ranging it has from its communication with the satellite and/or on-board telemetry.

\footnotetext{
${ }^{2}$ This section, and the rest of the paper, discusses collision avoidance in terms of an active satellite maneuvering to avoid a satellite-on-satellite collision, because that is the most complex and demanding case. Satelliteon-debris and debris-on-debris collisions also occur and are more common. Anytime an actor induces a change in orbit to avoid a potential conjunction, concerns about causing new conjunctions apply.
} 


\section{B. Conjunction Screening}

Service4Less regularly requests conjunction screenings for SpaceCube from one or more CAS providers. For example, it contracts with Collision Cruncher Corp. Since U. of Spaceland does not consider SpaceCube's orbital parameters to be sensitive, Service4Less provides the $\mathrm{O} / \mathrm{O}$ ephemeris to get a more accurate conjunction screening. Collision Cruncher Corp. then requests an updated catalog from one or more SSAs. For example, it may screen against SpaceCat, a free catalog operated by the Department of Commerce. Alternatively, it may use SpaceCat's competitor, the Space Service Syndicate, which is vertically integrated and performs both CAS and SSA roles. In response to the request from Collision Cruncher Corp., SpaceCat shares the catalog data derived from the sensors it owns or contracts to receive data from, as well as any other up-to-date $\mathrm{O} / \mathrm{O}$ ephemeris that other $\mathrm{S} 3$ s have provided to SpaceCat and marked as releasable. Collision Cruncher Corp. provides Service4Less with a set of conjunction data messages (CDMs) for conjunctions between SpaceCube and other RSOs. The particular parameters for screening are set either by Collision Cruncher Corp. by default or provided by Service4Less as part of the request.

Service4Less assesses the received CDMs, determining what events (if any) merit a potential collision avoidance maneuver, which can be cleared by analysis, and which are sufficiently far into the future that they can be safely monitored while awaiting further information. In our example, Service4Less determines there is a high interest event (HIE) between SpaceCube and SatCom1 which merits a collision avoidance maneuver. Service4Less looks up the S3 for SatCom1, sees that SatComCo acts as both the O/O and S3 for SatCom1, and sends a warning message with the CDM for the HIE.

If time permits, either Service4Less or SatComCo could contact an SSA provider to request tasking of additional sensors for better covariances associated with the position of either spacecraft, in case additional observations rule out a collision or reduce the magnitude required for a maneuver. Updated orbital information provided by the SSA provider can be shared with the other S3, based on the terms of allowed redistribution. In this example, we assume the new observations confirm that an avoidance maneuver is required.

\section{Collision Avoidance}

The two S3s, Service4Less and SatComCo then begin negotiations to determine who will move ${ }^{3}$. There are many potential ways to decide which spacecraft will move, based on factors such as operator preference, cost, risk, random chance, etc. The decisions are guided by technology and policy, with implications for system autonomy and conditions for which human-in-the-loop control is required. With some

\footnotetext{
${ }^{3}$ It is possible that the two S3s might disagree if a maneuver is required in which case the one who believed it was necessary would maneuver itself. There may eventually be regulatory standards to guide such decisions, but here it is assumed that all actions are voluntary.
}

mechanisms, it may make sense for both providers to generate their preferred maneuver prior to beginning negotiations, particularly if there is a temporal dependency relevant to the negotiations (i.e. SatCom1 could comfortably wait until $8 \mathrm{hrs}$ before the conjunction to maneuver, but SpaceCube only has access to drag-based orbital control and would need at least 36 hours to safely avoid a collision if it had to maneuver into a high drag state).

After exchanging proposed maneuvers and agreeing on a maneuver (assume by SatCom1), that maneuver would be validated by a CAS to ensure that it does not result in a secondary conjunction ${ }^{4}$ between the two satellites or passive debris $^{5}$. The maneuver would (with consent from both S3s) also be disseminated to the STM system overall to ensure it does not result in a conjunction with any third-party spacecraft. This broadcast message may be deliberately fuzzed to decrease precision, if either $\mathrm{O} / \mathrm{O}$ had concerns about proprietary sharing of orbital information. Other S3s would then have a certain period of time to indicate concerns with the proposed maneuver, after which the disseminating SatComCo would assume no objection. The time period for replies, as well as the epoch for the proposed maneuver would be included in the disseminated message.

Either concurrently or subsequently, depending on S3 and $\mathrm{O} / \mathrm{O}$ preference or any temporal constraints, O/O SatComCo will need to approve the maneuver (either autonomously or at the human level). Once approved, its S3 branch would disseminate a notice of the planned maneuver and timing to the broader system. It is also possible that a sophisticated $\mathrm{O} / \mathrm{O}$ might counter the maneuver proposed by its own S3, with an alternative to minimize mission impact or for other reasons. If the $\mathrm{O} / \mathrm{O}$ proposes another maneuver, the $\mathrm{S} 3$ would then go through the same process to clear that maneuver instead. After maneuver completion, there would be another system-wide message. One or both S3s might task an SSA, or simply request updated positional information, to verify the maneuver was performed and the spacecraft are in their expected positions.

\section{Enabling Autonomy Within the STM ARCHITECTURE}

We describe below a few initial, but representative considerations for leveraging autonomy to make the STM architecture more structured, efficient, flexible and open. As before, we continue to use physical collisions to illustrate the considerations, because it produces the most long-standing damage to the safe use of space. However, the assessment, strategic deconfliction, and data exchange ideas presented are applicable to other domains within STM such as radio interference.

\footnotetext{
${ }^{4} \mathrm{By}$ secondary conjunction, we mean a conjunction that results from an attempt by one of the two satellites maneuvering to avoid the initial primary conjunction. The differences between primary, secondary, and tertiary conjunctions and collisions is discussed further in section VI.

${ }^{5}$ There is a CCSDS message standard under development for spacecraft maneuvers. If approved, it would likely be used here.
} 


\section{A. Collision Assessment}

Following the identification of conjunctions, considerable decision-making occurs to assess potential collisions and determine whether a conjunction can be safely disregarded, needs to be monitored further, or if a collision avoidance maneuver is necessary. Additional autonomy during each of these phases would be helpful, initially to rule out easy cases and allow humans to focus on higher interest events, and eventually to augment or even surpass human decision-making ${ }^{6}$.

Depending on the $\mathrm{O} / \mathrm{O}$ and nature of the conjunction, there are multiple techniques that can be used to rule out a collision, with varying levels of computational or analytic effort required [5, slide 18-28]. Autonomy will contribute to determining which techniques to apply, and selecting cases for human observation. S3s also need to decide when to task additional observations, which can help confirm or rule out the need for a collision avoidance maneuver, but typically results in a delay to taking action and may involve a financial cost. Autonomy could help detect patterns to determine when additional observations are likely to be helpful, decide between different possible sensors or observation windows, weigh the expected benefit of potential observations against the cost of acquiring them, and if delay will reduce potential avoidance maneuvers or increase $\Delta \mathrm{V}$ requirements. Once a maneuver is determined to be necessary, autonomy could help identify the best maneuver(s) based on various operator, regulator, or system-defined characteristics and facilitate negotiation between S3s during a satellite-on-satellite conjunction.

\section{B. Collision Avoidance - Who Moves?}

Setting rules for which operator moves to avoid a high risk conjunction is beyond the scope of the architecture, but an important question for an operational system. While, under certain situations maneuver generation would be handled exclusively by the $\mathrm{S} 3$ of the more private $\mathrm{O} / \mathrm{O}$, the maneuver could potentially be conducted by either spacecraft ${ }^{7}$. Other factors that could influence which spacecraft moves include policy, technical parameters (such as the relative timing of potential maneuver windows for each craft), whether one $\mathrm{O} / \mathrm{O}$ and its S3 trusts the technical competency of the other S3 and craft to conduct an avoidance maneuver (a large communications satellite $\mathrm{O} / \mathrm{O}$ might prefer to move its satellite rather than trust a university-flown small satellite to correctly maneuver). A few high level methods to autonomously determine which satellite should move are described below. Ideally, standards would be developed by an inclusive group of $\mathrm{O} / \mathrm{O}$ s and other stakeholders.

a) Rules-Based System: There would be a set of agreedupon right-of-way rules that dictate which $\mathrm{O} / \mathrm{O}$ should move.

\footnotetext{
${ }^{6}$ Large O/Os operate sophisticated flight dynamics teams with rigorous processes, that may already incorporate some level of autonomy. Further consultation with such stakeholders, should they be willing to publicly share their practices, will be invaluable to defining the future direction of this work.

${ }^{7}$ In practice, we imagine that the more secretive $\mathrm{O} / \mathrm{O}$ would conduct a maneuver rather than create a maneuver for the other craft, to avoid sharing trajectory information and seeking consent from the other S3.
}

A useful analogy can be drawn from the rules governing right-of-way for sailboats, which tend to prioritize the less maneuverable craft, but also include arbitrary prioritization as necessary -i.e. leeward (downwind) over windward (upwind) and starboard tack over port tack. Regardless of the rules, naturally, a craft should still seek to avoid a collision. A rules-based system needs to be carefully designed to minimize otherwise counterproductive system-exploiting behavior by $\mathrm{O} / \mathrm{Os}$ seeking to externalize the cost of collision avoidance onto other crafts. A rules-based system also needs widespread and unified adoption to be successful. It is not self-enforcing (except to the extent actors see it in their own interest to comply) and may be of limited effectiveness without any accompanying method for liability enforcement ${ }^{8}$.

b) Dual-Maneuver Implicit Cost Split: Both craft would maneuver in a way that poses an equitable cost in consumables, mission disruption, or some other agreed-upon cost parameter. The advantage of such an approach is that it results in an implicit split of cost without requiring monetary transfer or other compensation. It is complicated by difficulties in quantifying mission disruption and consumable valuation, need for extensive communication to mutually plan maneuvers, a larger number of failure modes, and automation challenges.

c) Auction-Based System: O/Os would offer to move their craft to avoid a collision for a certain price to be paid by the other conjuncting craft. The other $\mathrm{O} / \mathrm{O}$ would be free to counter-offer another cheaper price, and the bargaining would continue until a lowest price was reached. Rates could potentially go up if new observational information changed the situational awareness of either operator. This system can be automated with relative ease. It would ensure that the craft with the lower cost of avoidance would maneuver and be compensated. In the absence of other policy enforcement mechanisms, small craft could potentially extract rents by pricing avoidance at an amount just below the cost of avoidance for large satellites, and risks incentivizing collision-seeking behavior by small maneuverable spacecraft against large riskaverse operations. Operationally, it would implicitly favor richer operators, and may be problematic if a conjunction occurs between satellites overseen by states that do not permit financial transactions between their respective citizens, or that have sanctioned a particular operator.

d) Resource-based System: The operator who would experience a lower cost of avoidance should conduct the maneuver. Cost could be assessed in terms of propellant usage, financial cost, or other mechanisms. If successful, this approach ensures the minimum total cost of mitigation. Challenges include ensuring accurate cost signaling by owner/operators, agreeing on cost definition, and the fact that such a system considers a lower absolute cost of movement, but not the significance of that cost to the operator (as a fraction of their

\footnotetext{
${ }^{8}$ Space liability is an interesting and complex topic in international space law, but is largely unsettled due to limited precedent.
} 
budget). It may also be difficult to equate cost metrics across very different types of satellites, and challenging to automate.

e) Last-Minute: Both crafts continue on their trajectories, in spite of being aware of an impending conjunction, until one decides to maneuver and notifies the other. While simple and self-enforcing, it is disadvantageous to the more riskaverse operators, and increases the likelihood of late or no mitigation maneuvers. It may also increase consumable cost by encouraging operators and their S3s to wait until closer to a potential conjunction to move (in hopes the other craft will move), increasing the $\Delta \mathrm{V}$ cost of their ultimate maneuver compared to if they had acted earlier. While last-minute avoidance might evolve in the absence of another system, it is not a system that policy-makers or operators should seek to promote.

\section{Catalogs - Centralized vs. Distributed?}

A basic architectural question with profound system consequences and implications for autonomy mechanisms is whether the architecture should have a single authoritative master catalog of space objects, or if it should support multiple non-authoritative catalogs. In case of the former, the supplier of that catalog would be responsible for ingesting, filtering, and deconflicting between multiple sources of potentially contradictory information. In case of the latter, they contain incomplete or differing information about object locations, with potential complications for conjunction screening and collision avoidance if the S3s involved are relying on different (and limited) sets of information ${ }^{9}$. For the sake of defining the architecture, it is not necessary to define who will develop, own, and/or operate the catalog(s). Options might include government entities, private contractors to government, companies, non-profit associations, and international organizations.

There at least four possible permutations of catalog architecture including a (1) single authoritative catalog (similar to U.S. air traffic control and UTM), (2) a small $\mathrm{N}$ number of high quality catalogs (with $\mathrm{N}$ small enough for CAS providers, $\mathrm{S} 3 \mathrm{~s}$, and $\mathrm{O} / \mathrm{Os}$ to manually or semi-manually decide to use a particular catalog and incorporate it into their workflow), (3) a large $\mathrm{N}$ number of catalogs (where verification and validation would need to be automated or mostly-automated), or (4) a small $\mathrm{N}$ or large $\mathrm{N}$ set of catalogs combined with algorithms to develop a single decentralized consensus.

1) Single Authoritative Catalog: With a one catalog approach, a single body within the architecture would be responsible for maintaining a catalog of RSOs. It would choose how to incorporate various sources of SSA information and fuse that data into a single cohesive picture of the orbital environment. The catalog custodian could be subject to various forms of oversight, depending on the type of owner (commer-

\footnotetext{
${ }^{9}$ It is also important to note that at an architectural level, the decision to have one or multiple catalogs is separate from the question of what SSA sources catalog(s) should ingest data from, or what SSA products should be derived from that data.
}

cial, government, or non-profit $)^{10}$. The Analytical Graphics, Inc. (AGI) Center for Space Standards and Innovation argues that existing catalog information is insufficiently accurate or precise to provide a basis for actionable SSA and STM across all relevant orbits [6, p. 7-10].

A one catalog approach has several key advantages. Because data is verified and validated at catalog ingestion, there is largely one set of truth about what objects are in orbit, where they are, and who owns them ${ }^{11}$. Rather than S3s and CASs being tasked with SSA source curation, validation, and data fusion, it would be done by the catalog. Having one source of truth simplifies maneuver coordination and helps avoid scenarios where different satellite O/Os make maneuvers based on different spatio-temporal understandings that either result in an unforeseen collision, or produce a collision with a third party operating with a different spatio-temporal understanding. Having a single catalog at an actionable level of accuracy is arguably also a prerequisite for any kind of governmentmandated control (as opposed to a $\mathrm{S} 3$ and $\mathrm{O} / \mathrm{O}$ coordination mandate). The lack of a single unified understanding increases risk for S3s, who might then seek or require some form of indemnification from their customers or government, which in turn encourages laxer practices by $\mathrm{S} 3 \mathrm{~s}$ interested in catering to less risk-averse O/Os.

On the other hand, having one catalog limits recourse if that catalog does not perform at the level desired by stakeholders, who will be limited in their ability to push improvements to that system or develop sustainable alternatives. Similarly, various stakeholders may have different, somewhat incompatible requirements for the system, and a single catalog may not meet all needs. This may particularly be a concern if the system operates with a funding model that passes costs through either directly to O/Os or S3s who serve them. Some O/Os may require and being willing to pay for exquisite positional accuracy due to operating expensive spacecraft in congested orbits, while others would be satisfied with relatively coarse information to avoid gross collision risk at minimal cost. It also means that the catalog custodian, subject to whatever checks are put in place, would make decisions about which sources of SSA data to procure, potentially including commercial decisions about what data to purchase at what prices. This may result in a commercial conflict of interest if some SSA sources are operated by the catalog provider while others would need to be purchased from the operator's commercial competitors. A single system would also need to tailor clear rules for access, acceptable use, and information sharing. It would either need to provide SSA products adequate to meet the needs of all

\footnotetext{
${ }^{10} \mathrm{~A}$ commercial entity could be regulated similar to other natural monopolies such as utility companies, a government body would be subject to administrative procedure requirements, as well as agency and congressional oversight, and a non-profit would be subject to management by a board of key stakeholders.

${ }^{11}$ There will likely always be operators unwilling to cooperatively share data with the catalog, either for commercial competition or national security reasons. The catalog would need to develop ways to interact with these entities and decide how much information to provide to them, in what formats, and with what conditions of use.
} 
users, or have acceptable procedures to provide information to third parties, without losing the trust of $\mathrm{O} / \mathrm{O}$ s sharing their sensitive, proprietary information. From a cybersecurity risk perspective, a single system presents a single target and point of failure, should that system be compromised. While in the case of a short, rapidly detected breach, specific positional information would only be of limited use (as propagation errors diverge), there is a significant proprietary risk if satellite geometry, maneuver planning, and spectrum/RF information is compromised.

2) Small N Number of Catalogs: The current de facto state of STM is a small $\mathrm{N}$ set of catalogs, with various providers seeking to differentiate themselves and their offerings from the free Joint Space Operations Center (JSpOC) catalog and conjunction warnings ${ }^{12}$. A small $\mathrm{N}$ number of catalogs provides greater flexibility and accountability than a single authoritative catalog, but still allows for a manual or semi-manual assessment and interface between the catalogs, stakeholders who would provide data to the system, and downstream users of the systems. Multiple catalogs with overlapping RSO coverage derived from different sensor networks could also potentially provide a robust mechanism for cross-validation of data quality. A small $\mathrm{N}$ set of catalogs is an inevitable consequence if catalog operators design their catalogs with only capability to ingest data from a limited number or type of source. A small $\mathrm{N}$ is distinguished from a large $\mathrm{N}$ because when $\mathrm{N}$ becomes sufficiently large, it will require standardized, off-theshelf, interoperable interfaces with autonomous integration of quality-assessed SSA data. Large $\mathrm{N}$ offers operators additional flexibility, choice, and reduced transaction costs associated with discovering, assessing, validating, verifying, fusing, and negotiating for access to each catalog, at a greater initial cost in ensuring interoperability and automation.

Existing non-government providers include AGI, ExoAnalytic, LeoLabs, and the Space Data Association, each of which also integrates aspects of SSA collection and conjunction analysis. To the authors' knowledge, there is no third-party certification of these catalogs, rather individual customers evaluate their offerings and make decisions regarding which, if any, to incorporate into their collision avoidance and other SSA analysis. It is useful to again draw a distinction between non-cooperative data, which generally can be widely shared subject to only commercial concerns, and operator-furnished maneuver or meta-data, which is critical for accurate modeling and prediction, but where proprietary concerns can in many cases prevent consent for public dissemination.

The small $\mathrm{N}$ approach is compatible with a regulatory scheme that attributes responsibility for collision avoidance to $\mathrm{O} / \mathrm{Os}$ or $\mathrm{S} 3 \mathrm{~s}$, without attempting to influence risk tolerance, but works less well if the regulatory scheme attempts to compel greater risk-aversion among risk-tolerant operators. If an operator has a choice among multiple catalogs, it may simply choose the cheapest one (or the cheapest S3, which

\footnotetext{
${ }^{12}$ Even though multiple catalogs and providers exist, JSpOC is by far the most dominant provider of SSA information, especially in LEO.
}

draws on the cheapest catalog), or potentially cherry pick between catalogs to find data that shows its satellite will not collide.

Multiple catalogs also increase complexity of collision avoidance maneuver coordination, since S3s will need to deconflict state information for the spacecraft in question, as well as potentially impacted third-party spacecraft, to screen for maneuver induced conjunctions. The most accurate orbital determinations require cooperative maneuver information from $\mathrm{O} / \mathrm{Os}$, and providing this information to more than one trusted third party expands the required trust envelope. If only one catalog receives this operator-furnished information, and others simply provide additional non-cooperative tracking, the situation becomes similar to the one catalog case, as the catalog with operator-furnished information will simply need to come to agreements to ingest observations from the other catalogs (since O/Os may be sharing-averse).

3) Large $N$ Number of Catalogs: A large $\mathrm{N}$ number of catalogs envisions multiple catalogs, where $\mathrm{N}$ is so large that it is not feasible for $\mathrm{S} 3 \mathrm{~s}$ or $\mathrm{O} / \mathrm{Os}$ to independently or manually devote effort to interfacing with particular catalogs or assessing the quality of the data provided. Instead, interfaces would need to be highly standardized and operators would need to develop robust and autonomous verification and value determination algorithms to evaluate potential sources of data and choose which to ingest. The large $\mathrm{N}$ case features greater initial technical complexity, but results in a market with reduced switching and transaction costs, where S3s and CASs interface with catalogs as a main feature in building their SSA. Market forces would result in significant competition among catalog providers and keep prices low, at least so long as the market does not tend toward consolidation to become a natural monopoly.

The large $\mathrm{N}$ case is characterized by significant difficulty in maneuver coordination and determining how to handle sensitive data from providers. The secure multiparty computation cryptographic technique referenced in the next subsection might provide a way to share sensitive state information for the purpose of conjunction analysis, without revealing the underlying information.

4) N Number of Catalogs with Algorithmic Consensus: This option conceptualizes the creation of a consensus metacatalog among multiple catalogs (small or large $\mathrm{N}$ ) in a robust and distributed manner. Catalogs would share their information about RSOs, and an autonomous algorithm would pool data, resolve conflicts (i.e. where catalogs place objects in mutually inconsistent orbits), and determine positional, ephemeris, and covariance information for each object. The advantage of this approach is that it can allow the development of a single authoritative catalog, with associated benefits for collision avoidance maneuver coordination, without requiring a single organizational catalog.

There would be multiple challenges for developing such a system, the first being if it is achievable and robust from a technical perspective. An algorithm would need to meet very high standards for reliability and security, and probably be able 
to autonomously identify and reject deliberately harmful information submitted by catalogs. The second relates to sharing of proprietary $\mathrm{O} / \mathrm{O}$ information necessary to produce the metacatalog. Multiparty computation (MPC) provides a mechanism to securely share inputs for computations in a way that shields the inputs from other parties, while publicly disclosing and confirming the result of a computation. MPC may provide a useful technique for such a meta-catalog, but challenges associated with computational complexity remain [7]. Third, funding allocation is tricky, but solvable. The meta-catalog would need to provide a mechanism to apportion funding for contributing catalogs under an algorithmic system. Since data is automatically contributed, S3s and O/Os would no longer make funding decisions. Instead, we need an algorithm that weighs the value of the contributions, perhaps based on usefulness for collision detection or number of observations, or accuracy against a randomly selected and exquisitely known set of objects. The authors are not certain if an algorithmic consensus mechanism is technically feasible, but include it for future consideration.

\section{AdDressing The Challenges of STM Autonomy ApPlied to Collision AVOIDANCE}

The current state of SSA is a small $\mathrm{N}$ number of catalogs. Evolution to a single catalog or $\mathrm{N}$ catalogs with consensus (behaving like an integrated catalog) may eventually be desirable, but is premature until SSA information \& sensors, data product needs, and derived SSA product generation methods solidify to a level that data can be pooled to meet the needs of S3s \& $\mathrm{O} / \mathrm{Os}$ for actionable information. In the meanwhile, designing a method that permits feasible system-wide deconfliction of potential conjunctions within a multi-catalog architecture is necessary, because collision avoidance is a non-negotiable requirement for an STM system. This section explores the considerations of operating such a system as algorithmically and autonomously as possible. The software research platform proposed for development at NASA ARC will be critical to assessing the feasibility of these concepts.

\section{A. Conceptualizing Types of Conjunctions}

When an active satellite moves to avoid a potential conjunction, it is acting to mitigate three potential sets of conjunctions: the primary conjunction that motivated the maneuver, any secondary conjunctions between the manuevering satellite and the object included in the initial conjunction, and any tertiary conjunctions between the maneuvering satellite and other RSOs. While secondary and tertiary conjunctions will likely only be screened for some limited time period into the future (subject to limits in orbit propagation accuracy), the maneuver will not be fully successful if it does not clear all three sets of conjunction constraints over this time period, thereby requiring another maneuver shortly thereafter.

\section{B. Collision Avoidance Scenarios as a Function of Data Availability}

Before discussing strategies for collision avoidance with multiple catalogs, it is important to note what data each S3 will have and what data is not necessarily shared. All operators will have access to the free catalog of government-provided SSA information, primarily derived from non-cooperative observations from the Space Surveillance Network, but potentially also from $\mathrm{O} / \mathrm{O}$ ephemerides when those O/Os have consented to publication, or from commercial or other SSA observations purchased by or donated to the government SSA catalog. There may also be non-cooperative observations from commercial or private sources, that may be shared with a limited set of users or not shared at all. Some level of filtering may be necessary to determine if observations from a particular source are in conflict with another, or to generate collision avoidance maneuvers based on a worst case scenario, or that avoid multiple possible conjunction paths for secretive or indeterminate SSA.

Differences between non-cooperative observations and $\mathrm{O} / \mathrm{O}$ ephemeris information can be very substantial, especially if a non-cooperative orbit is propagated based on a pre-maneuver or mid-maneuver observation. The architecture assumes and exploits the fact that the S3 representing a particular $\mathrm{O} / \mathrm{O}$ will have access to this information, and will know its orbit authoritatively and correctly, even if it differs from noncooperative observations. It further assumes that S3s and $\mathrm{O} / \mathrm{Os}$ are incentivized to avoid collisions and will act accordingly, even if they are subject to limitations on sharing $\mathrm{O} / \mathrm{O}$ ephemerides.

A successful strategy for system-wide collision avoidance needs to address several scenarios: (1) when accurate positions of both spacecraft are known to both S3s, (2) When accurate information for one satellite is known to both $\mathrm{O} / \mathrm{Os}$, but accurate information for the other is only known to its own O/O (and the first does not necessarily know it has an inaccurate trajectory for the second), (3) When both operators have known states for their own spacecraft, but inaccurate information for the other spacecraft (neither necessarily knows this).

There can also be variation in the level of divergence between the information known to the $\mathrm{S} 3$ representing an $\mathrm{O} / \mathrm{O}$ and that available to the other S3 from SSA provider(s). In the case of a small divergence, resulting from routine stationkeeping maneuvers, a sufficiently sized screening volume during conjunction screening may be sufficient to accommodate the error. In the case of a larger maneuver, where an inclusive screening volume is large enough to produce an unacceptable number of false positives, only the $\mathrm{S} 3$ and $\mathrm{O} / \mathrm{O}$, or SSA/CAS providers who they have shared information with, will have the potential to accurately screen for conjunctions. Since at least some $\mathrm{S} 3 \mathrm{~s}$ and $\mathrm{O} / \mathrm{Os}$ will be unwilling to fully share this information, the architecture must rely on them to perform conjunction analysis in the case of a large maneuver, and disseminate an authoritative conjunction assessment. In the rare case where two spacecraft both engage in large maneuvers simultaneously, there is no way (to the authors' knowledge) to ensure a collision is avoided without at least one of them sharing positional information with the other, with sufficient resolution to rule out a conjunction (but not 
necessarily to precisely describe its own position). Information could potentially be shared as an input to a secure MPC to enable screening without revealing the underlying trajectory information. Such computations involve significantly increased time and resources as compared to non-encrypted techniques, and may only be viable in the case of pre-identified areas of concern, rather than allowing all against all screening in the case of large maneuvers.

\section{Levels of Data Sharing}

$\mathrm{O} / \mathrm{Os}$ vary in their willingness to share ephemeris information. One can imagine a (non-exhaustive) scale of comfort in sharing such information. The scale below ranges from most open to least open to sharing. It is meant to be conceptual to help address information needs in the context of mitigating primary, secondary, and tertiary conjunctions. Interviews and other engagement with existing $\mathrm{O} / \mathrm{Os}$ would be necessary to better understand and categorize the willingness of actual $\mathrm{O} / \mathrm{Os}$ to share orbital information. In the absence of $\mathrm{O} / \mathrm{O}$ provided data, S3s and other actors in the STM system have to fall back on surveillance (non-cooperative) measurements.

- Level 5 - Global publication of orbital information: $\mathrm{O} / \mathrm{Os}$ are willing to publish ephemeris information to the STM system regularly for use by others and ingestion into any SSA that chooses to use the data.

- Level 4 - Legal protected/trusted environment: O/O are willing to publish ephemeris information, but only within the context of an environment that places legal and technical restrictions on use, to address the O/O's concerns about competitive advantage or other consequences of sharing.

- Level 3 - Particularized data for a certain conjunction: $\mathrm{O} / \mathrm{O}$ is willing to provide information for limited period of time, upon request, in order to screen or clear a particular conjunction, but is unwilling to provide more general access to such information. It may insist on the use of MPC to minimize data disclosure.

- Level 2 - Confirm or deny a threat of potential collision: some O/Os may be unwilling to provide even limited orbital information, but might be willing to screen a requester's spacecraft orbit against their known data, and either confirm or rule out the need for action.

- Level 1 - No sharing: Some O/O may be unwilling to share $\mathrm{O} / \mathrm{O}$ ephemeris information in the absence of legal compulsion to do so.

\section{Automating Collision Avoidance with Varying Data and Multiple Catalogs}

Actors in the system should use the best accuracy data available to them, and policy-makers may wish to put in place incentives to encourage greater data sharing. Even if the STM architecture would be able to avoid a conjunction with more limited data, more data may help reduce false positives, or conserve propellant for required maneuvers. The following items describe informational needs, and possible strategies at each step in the collision avoidance chain. a) Initial Screening for Potential Conjunctions: CASs generate a list of conjunctions using the best information available to them. Screening parameters should be set generously enough to not require $\mathrm{O} / \mathrm{O}$-shared ephemeris data to flag potential conjunctions. This is possible with no $\mathrm{O} / \mathrm{O}$ sharing but enhanced by $\mathrm{O} / \mathrm{O}$ data. Since large maneuvers are not known to the system, initial screening cannot address them without CAS access to maneuver information.

b) Collision Assessment: The S3 would review the potential CDMs, autonomously determining (1) when it would be necessary to act on each of them, (2) when to task additional sensors to observe potential threats, (3) what constitutes a threat that requires warning of the other S3, (4) when to generate collision avoidance maneuvers, (5) what those maneuvers should be, and (6) at what point in this process should humans be brought into the loop and when these steps can be automated. As described previously, this decision chain is one of the major components where autonomy will be critical to enabling the STM architecture.

c) Primary Conjunction Mitigation: Both S3s need to understand the trajectory of the other spacecraft well enough to determine if there is an unacceptable risk of collision. Following up on the generalized screening conducted by the CAS, the S3s would contact each other in an attempt to verify their understandings of the trajectory of both spacecraft ${ }^{13}$, and best estimate the time of closest approach. If only one of the two S3s assesses that there is a risk of collision, it would then unilaterally direct its spacecraft to be the one that moved, but would still share as much data as it was willing to disclose.

d) Secondary Conjunction Mitigation: Either both operators need to know the position of the other craft with sufficient accuracy to ensure that a secondary conjunction does not occur, or both operators need to trust one of the $\mathrm{S} 3 \mathrm{~s}$ to conduct coordination on behalf of both spacecraft. If only one spacecraft moves to avoid a potential conjunction, both S3s trust the S3 of the moving spacecraft, by default. If the position of spacecraft $A$ is well known by both $S 3 \mathrm{~s}$, level 2 information sharing may be sufficient, with the S3 of Spacecraft B either clearing a maneuver proposed by the S3 of Spacecraft A, or simply confirming to the S3 of Spacecraft A that it will maneuver in a way to clear the conjunction and that Spacecraft A should maintain its existing or an agreedupon trajectory. If neither S3 has well characterized positional information for the other craft, either larger margins will be necessary to avoid a collision or at least level 3 information sharing is required (perhaps using MPC).

e) Tertiary Conjunction Mitigation: The key distinguishing fact about tertiary conjunction mitigation is that the maneuvering craft does not know with which potential operators

\footnotetext{
${ }^{13}$ This verification process is limited by willingness to share information. Both S3s could declare their willingness to share information. If both S3s are willing to share positional information, they can simply conduct an open comparison to verify their mutual positional understanding. If unacceptable to one party but acceptable to the other, the more private O/O's S3 could take responsibility for collision assessment and avoidance without necessarily revealing its own orbit ephemeris. If both demand restrictions, collision assessment would default to using MPC or a mutually trusted third-party.
} 
it might enter into a conjunction (due to differences between the information its S3 has and that held privately by other actors). The S3 for the maneuvering object would check the proposed maneuver against the best SSA information available to it about all RSOs. This would clear most objects, but not those of maneuvering satellites with undisclosed ephemeris. If the maneuvering $\mathrm{O} / \mathrm{O}$ is willing to share its planned trajectory globally (level 5), it can simply declare a planned maneuver to the full system and wait for any objections (as in the example in section IV), which places the burden for screening the proposed maneuver on those S3s who represent O/Os unwilling to share $\mathrm{O} / \mathrm{O}$ ephemeris information. If the maneuvering $\mathrm{O} / \mathrm{O}$ is unwilling to provide exquisite orbital information, it could provide a deliberately fuzzy track, and engage in follow-up one-on-one deconflictions with any other O/Os that declare a potential conjunction with that track, potentially again using MPC. If it is unwilling to share any information (level 1), and the primary maneuver is small, it may simply be captured within existing conjunction screening margins by other operators. If the primary maneuver is large, and the $\mathrm{O} / \mathrm{O}$ is unwilling to declare it, it is difficult for the STM system to assure tertiary non-conjunction. A subset of undeclared large maneuvers could be cleared by placing bounding assumptions on the movement of third party satellites (i.e. it would be physically impossible for this craft to maneuver into an orbit that would produce a conjunction with my satellite's new trajectory). Unfortunately, the subset that can be cleared is also the subset least likely to pose a risk of conjunction. Clearing the remaining conjunctions of concern would require access to $\mathrm{O} / \mathrm{O}$ ephemeris or other exquisite orbital trajectory information for those spacecraft.

\section{E. What About Non-Participants?}

At each of these steps, participating S3s will only be able to avoid non-participants of which they or their SSA providers have accurate knowledge. The system does envision a public API to allow non-participants to access the STM architecture, subject to limitations as necessary, for research, reporting, development of new products, or for non-participant use. Oltrogge describes a category of "Willing Non-Contributor Operators (WNCOs)" who are interested in preventing collisions, but are unwilling to provide data into an STM system (whether for commercial, national security, or other reasons [6, p. 17]. In our proposed architecture, these entities would maintain an obligation to avoid collisions, including with maneuvering spacecraft. A risk exists for collision between maneuvering spacecraft unwilling to disclose their locations and WNCOs, but these conjunctions would by definition need to be handled outside the system.

Investigation using the STM software research platform would inform what constitutes a typical threshold for a small vs. large maneuver, how much of a threat exists for potential collision between two maneuvering spacecraft, and if policy mandating the disclosure of large maneuvers is necessary to provide assurance of orbital deconfliction in an openarchitecture STM system.

\section{NeXt Steps And Ways to Participate}

As a next step, the NASA STM group plans to continue presenting the proposed architecture to potential stakeholders to further refine the concept. Once the concept has been matured and stabilized, work will begin to define APIs, roles, and functions, as well as the details of the varying technology capability levels. Concurrent with the definition work, we have begun on work to develop the software research platform, as well as set up a physical laboratory and visualization environment at the NASA Ames Research Center. The team also plans to develop and test tools for automating of various components of conjunction assessment, building on both predeveloped in-house code [8] and existing tools such as AGI's STK AdvCAT module and the European Space Agency's DRAMA tool. The team actively invites feedback from O/Os and other STM stakeholders to help inform the proposed STM architecture, and to collaborate in the development and testing of the proposed software research platform.

\section{ACKNOWLEDGMENT}

The authors thank the NASA UAS Traffic Management Team for their work to develop the UTM concept from which this concept draws inspiration.

\section{REFERENCES}

[1] National Space Council, "Space Policy Directive 3: National Space Traffic Management Policy," 2018. [Online]. Available: https://www.whitehouse.gov/presidential-actions/space-policydirective-3-national-space-traffic-management-policy/

[2] A. Abraham, "GPS Transponders For Space Traffic Management," Colorado Springs, CO, 2018. [Online]. Available: https://aerospace.org/paper/gps-transponders-space-traffic-management

[3] Federal Aviation Administration, "Unmanned Aircraft System (UAS) Traffic Management (UTM) Concept of Operations Version 1.0," Federal Aviation Administration, Tech. Rep., 2018. [Online]. Available: https://utm.arc.nasa.gov/docs/2018-UTM-ConOps-v1.0.pdf

[4] A. S. Aweiss, B. D. Owens, J. L. Rios, J. R. Homola, and C. P. Mohlenbrink, "Unmanned Aircraft Systems (UAS) Traffic Management (UTM) National Campaign II," in 2018 AIAA Information Systems-AIAA Infotech @ Aerospace, 2018. [Online]. Available: http://arc.aiaa.org https://arc.aiaa.org/doi/10.2514/6.2018-1727

[5] D. L. Oltrogge and S. Alfano, "Satellite Operator Safety of Flight State of Health," in CNES International Conjunction Assessment Workshop, no. November, 2017.

[6] D. L. Oltrogge, "The "We" Approach to Space Traffic Management," in 2018 SpaceOps Conference, ser. SpaceOps Conferences. American Institute of Aeronautics and Astronautics, 5 2018. [Online]. Available: https://doi.org/10.2514/6.2018-2668

[7] B. Hemenway, W. Welser IV, and D. Baiocchi, "Achieving Higher-Fidelity Conjunction Analyses Using Cryptography to Improve Information Sharing," RAND Corporation, Tech. Rep., 2014.

[8] F. Yang Yang, B. Nelson, J. Aziz, R. Carlino, A. Dono Perez, N. Faber, C. Foster, C. Frost, C. Henze, A. G. Karacaloğlu, C. Levit, W. Marshall J. Mason, C. OToole, J. Swenson, S. P. Worden, and J. Stupl, "LightForce photon-pressure collision avoidance: Efficiency analysis in the current debris environment and long-term simulation perspective," Acta Astronautica, vol. 126, pp. 411-423, 9 2016. [Online]. Available: https://www.sciencedirect.com/science/article/pii/S0094576516301205 